\title{
Level Crossing in Toroidal On-chip Microcavities
}

\author{
Harald G. L. Schwefel ${ }^{1}$, Lan Yang ${ }^{2}$, Mark Oxborrow ${ }^{3}$, A. Douglas Stone ${ }^{4}$, Kerry J. Vahala ${ }^{5}$, and Tal Carmon ${ }^{6}$ \\ ${ }^{1}$ University of Erlangen-Nuremberg, Institute for Optics, , Günther-Scharowsky-Str. 1, Bau 24, 91058 Erlangen, Germany \\ ${ }^{2}$ Department of Electrical and Systems Engineering, Washington University, One Brookings Drive, St. Louis, MO, 63130 \\ ${ }^{3}$ National Physical Laboratory, Teddington, Middlesex, TW11 OLW, UK \\ ${ }^{4}$ Yale University, Department of Applied Physics, New Haven, CT 06520, USA \\ ${ }_{5}^{5}$ Applied Physics Department, Caltech, Pasadena, California 91125 USA \\ ${ }^{6}$ University of Michigan, College of Engineering, 2200 Bonisteel Blvd, Ann Arbor, MI 48109-2099, USA \\ tcarmon@umich.edu
}

\begin{abstract}
Level crossing between optical whispering-gallery modes is studied in toroidal microcavities. We photograph azimuthal and radial envelope patterns of crossed optical modes. We also investigate anticrossing between modes and polarization evolution.

(C)2009 Optical Society of America

OCIS code: (140.3945) Microcavities; (140.4780) Optical resonators;
\end{abstract}

Dielectric whispering-gallery cavities [1] have a set of discrete optical eigen-frequencies that can cross each other [2]. Here we tune the ratio between the major and minor-diameters of a dielectric toroidal cavity to allow crossing between modes (fig 1c). On crossing, modes resonate at the same frequency but with different wavelengths resulting in spatial (most noticeably azimuthal) beatnotes (fig1 a, d). Our experimental on-chip toroidal cavity is doped with erbium [3], where its IR $(1.5 \mathrm{~mm})$ composite-mode pattern is rendered visible through partial up-conversion into green light via 3-photon fluorescence [4]. We measure crossings between modes with azimuthal orders ranging from 3 to 18 , and observe modulations in the azimuthal (fig 1.a-e) as well as radial directions (fig 1.g-f).

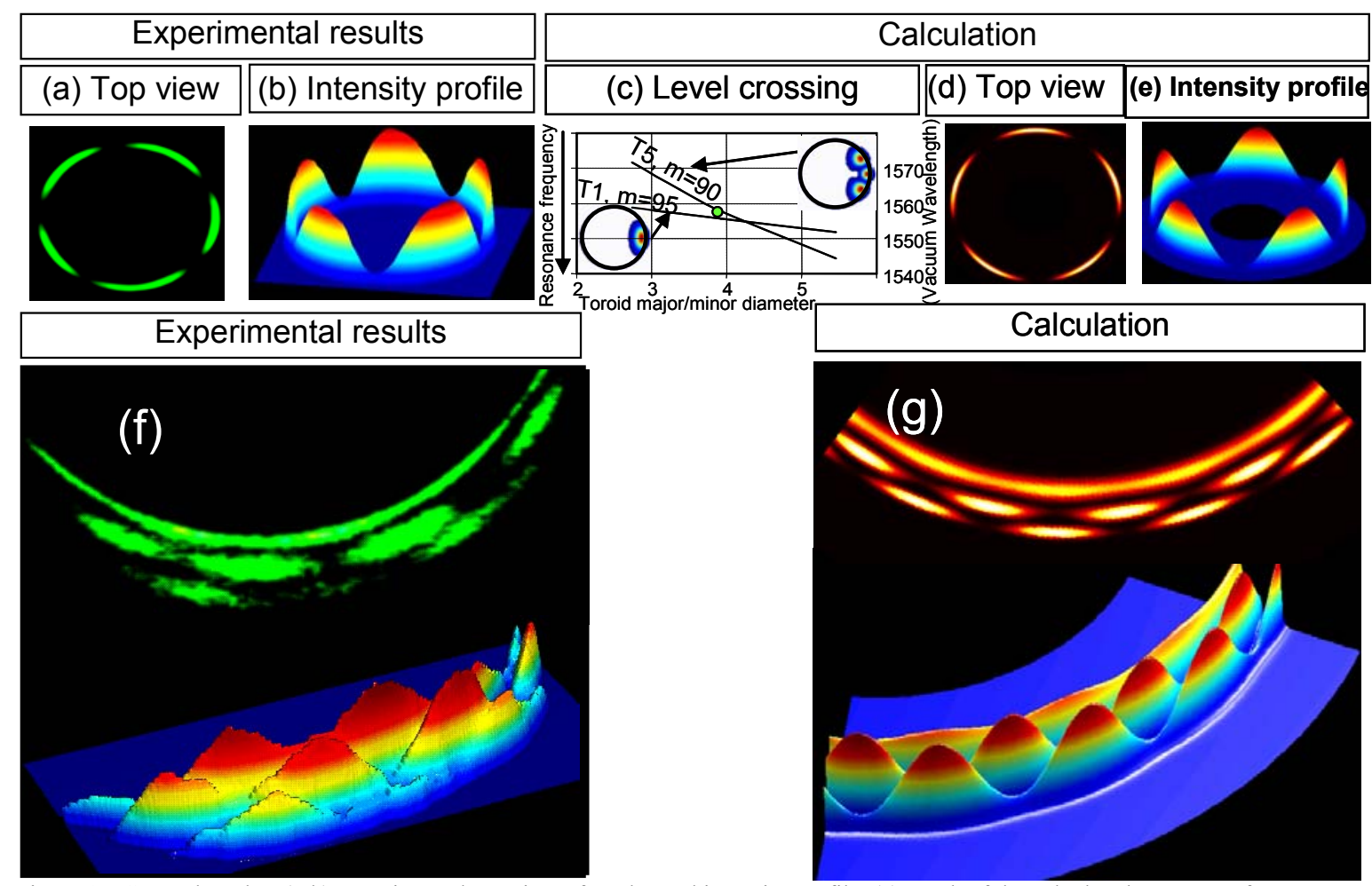

Figure 1: Crossed modes. (a-b) experimental top view of modes and intensity profile. (c) graph of the calculated resonance frequency, versus the ratio of the major and minor diameter, green circle in c represents the experimental parameters in a-b. (d-e), numerically calculated modes. (f-g), similar measured and calculated mode but with also radial interference pattern.

Anti-crossing between modes is calculated for modes of similar azimuthal wavelength but different transverse profile; in this case, complex polarizations are possible. In conclusion, we studied crossing (that we initially did not expect) between optical levels in an on-chip toroidal resonator. Complex patterns in the azimuthal, 


\section{CTuE2.pdf}

radial, and transverse directions are reported as well as composite polarizations. Beyond the phenomenon's fundamental geometrical interest, controlling the frequency difference between modes (from free-spectral-range to overlap) as reported here [5] is paramount in applications that require phase matching, such as third-harmonic generation [6] and stimulated Brillouin scattering [7].

[1] K. J. Vahala, Nature 424, 839 (2003).

[2] A. A. Savchenkov et al., Physical Review A 76, 023816 (2007).

[3] D. K. Armani, T. J. Kippenberg, S. M. Spillane, and K. J. Vahala, Nature 421, 925 (2003).

[4] L. Yang et al., Applied Physics Letters 86, 091114 (2005).

[5] T. Carmon et al., Physical Review Letters 100 (2008).

[6] T. Carmon, and K. J. Vahala, Nature Physics 3, 470 (2007).

[7] T. Matthew and T. Carmon, "Photonic MEMS vibrating at X-band rates (11GHz)", In Review (2008) 